\title{
Marco jurídico de las cooperativas en Cuba. Tracto histórico y realidad actual
}

\author{
Orestes Rodríguez Musa' \\ Universidad de Pinar del Río, Cuba
}

doi: http://dx.doi.org/10.18543/dec-14-2019pp17-44

Recibido: 25-10-2019

Aceptado: 18-11-2019

Sumario: I. Introducción. II. La legislación colonial. III. La Constitución de 1940. IV. La legislación socialista. V. El actual proceso de expansión de las cooperativas en Cuba. Limitaciones y perspectivas jurídicas. VI. Conclusiones.

Resumen: En el presente trabajo se valora el tracto histórico por el que ha transitado el marco jurídico de las cooperativas en Cuba, identificando las limitaciones y potencialidades ofrecidas a estas formas asociativas en cada período. Se hará especial énfasis en la realidad actual, iniciada con el perfeccionamiento del modelo económico cubano, donde se constata un proceso de expansión de las cooperativas hacia otros sectores de la economía nacional además del agropecuario. Las perspectivas jurídicas de este proceso experimental en curso también serán objeto de una valoración general, teniendo en cuenta las últimas novedades legislativas en la materia cooperativa, en especial la letra de la nueva Constitución de la República de Cuba de 2019.

Palabras clave: cooperativas, marco jurídico, tracto histórico.

Abstract: In this paper, the historical tract through which the legal framework of cooperatives in Cuba has been traced is valued, identifying the limitations and potential offered to these associative forms in each period. Special emphasis will be placed on the current reality, initiated with the refinement of the Cuban economic model, where a process of expansion of cooperatives to other sectors of the national economy besides agriculture is observed. The legal perspectives of this ongoing experimental process will also be subject to a general assessment, taking into account the latest legislative developments in the cooperative matter, especially the letter of the new Constitution of the Republic of Cuba of 2019.

Keywords: cooperatives, legal framework, historical tract.

1 Doctor en Ciencias Jurídicas. Profesor Titular del Departamento de Derecho de la Universidad de Pinar del Río, Cuba. E-mail: musa@upr.edu.cu 


\section{Introducción}

La promoción de las cooperativas, como medio para coadyuvar al bienestar ciudadano en Cuba, ha estado presente más de una vez en el pensamiento y la acción política de avanzada². La regulación jurídica de la figura no ha sido reflejo pasivo de la historia, sino que ha incidido dialécticamente en su evolución, en su estancamiento o en sus desviaciones prácticas.

Por consiguiente, se valorará a continuación, desde una perspectiva histórica, el marco institucional de las cooperativas en Cuba, a fin de identificar, en este recorrido histórico, las limitaciones y potencialidades ofrecidas por el ordenamiento jurídico a estas formas asociativas, para manifestarse como una herramienta integral de satisfacción de las necesidades socioeconómica de sus miembros y de la comunidad.

De cara a este propósito, pueden delimitarse tres períodos históricos fundamentales. El primero comienza con el traslado de instituciones peninsulares hacia la Cuba española a finales del siglo XIX, y se extiende hasta que los principios constitucionales de 1940 oxigenan la institución. Una segunda etapa que comienza con la aprobación de este texto constitucional y que paulatinamente desaparece cuando sus postulados son superados políticamente por el proceso revolucionario de 1959. El tercer período se observa con mayor nitidez tras la promulgación de la Constitución socialista de 1976 y se extiende hasta la recién iniciada actualización del modelo socioeconómico cubano, donde se busca redimensionar el sector cooperativo. Las limitaciones y perspectivas de este proceso experimental en curso serán también objeto de una valoración general en este trabajo, teniendo en cuenta la normativa vigente, en especial la nueva Constitución cubana de 2019.

2 Destacan en este sentido las referencias en el «Programa de la Joven Cuba» de Antonio Guiteras Holmes en 1934 [consultada en PICHARDO, H.: Documentos para la Historia de Cuba, t. IV (Primera Parte), Ed. Ciencias Sociales, La Habana, 1980, pp. 517525]; en el «Programa Constitucional» del Partido Revolucionario Cubano (Auténtico) en 1934 [consultado en ibidem, pp. 290-317]; en las «Bases para el Proyecto de Constitución» de la Unión Revolucionaria Comunista en 1939 [consultadas en idem, t. V, pp. 279 a la 305]; y en la «Historia me Absolverá» de Fidel CASTRO Ruz en 1953 [CASTRO Ruz, F.: La Historia me Absolverá, edición anotada por Álvarez TABío, P. y Alonso Fiel, G., Oficina de Publicaciones del Consejo de Estado y Ed. Pueblo y Educación, La Habana, 2005]. 


\section{La legislación colonial}

Durante la primera etapa existen normas que simplemente la reconocen, pero que no aspiran a atribuirle régimen jurídico propio. Tal es el caso del Código de Comercio de 1885 (trasladado a la isla en el siguiente año y aún vigente en parte), contentivo en su artículo 124 de una cláusula de excepcionalidad que expresamente las excluye de sus predios, a menos que se dedicaren a actos de comercio ${ }^{3}$, en cuyo caso no requerirían de un tipo societario especial, dada la amplitud con que se acoge la atipicidad en los artículos 121 y 122.

No es de extrañar que el Código no la regulara, en tanto en este cuerpo jurídico se asume el criterio del lucro en la determinación de la mercantilidad de las sociedades. A tono con ello, entiende el legislador en la Exposición de Motivos, que las cooperativas «...obedecen, ante todo, a la tendencia manifiesta (...) de asociarse los obreros con el único fin de mejorar la condición de cada uno... Y como no es el afán de lucro el que impulsa lo que se ha dado en llamar movimiento cooperativo, no pueden reputarse como mercantiles estas sociedades, mientras no resulte claramente de sus Estatutos o del ejercicio habitual de algunos actos de comercio que merecen aquella denominación ${ }^{4}$.

Si bien son válidos estos argumentos, también es cierto que a ellos subyacían razones ideológicas: las clases dominantes y hacedoras del Derecho en España, ante la posibilidad de que el movimiento obrero las utilizara para «constituir verdaderas sociedades de resistencia contra el capital» ${ }^{5}$, veía en ellas entes peligrosos que requerían de un instrumento de regulación más idóneo para el control político. En consecuencia, mediante la Ley de Asociaciones de 1887, dirigida a desarrollar el contenido político del derecho de asociación, se forzó un espacio legal a las cooperativas en su artículo primero ${ }^{6}$, donde apenas

3 El artículo 124 del Código de Comercio español [contenido en el Real Decreto de 22 de agosto de 1885, recuperado de https://www.boe.es/buscar/act.php?id=BOE-A-18856627, el 18 de junio de 2015], establece que «Las Compañías mutuas de seguros contra incendios, de combinaciones tontinas sobre la vida para auxilios a la vejez y de cualquiera otra clase y las cooperativas de producción, de crédito o de consumo, sólo se considerarán mercantiles y quedarán sujetas a las disposiciones de este Código, cuando se dedicaren a actos de comercio extraños a la mutualidad o se convirtieren en Sociedades a prima fija».

4 GADEA SOleR, E.: Evolución de la legislación cooperativa en España, Consejo Superior de Cooperativas de Euskadi, 1999, p. 129.

5 Idem.

6 Esta Ley establecía expresamente en el artículo 1 su aplicación a «las cooperativas de producción, de crédito o de consumo». Vid. Ley de Asociaciones española, contenida en el Real Decreto de 13 de junio de 1888, recuperada de https://www.boe.es/buscar/, el 18 de junio de 2015. 
existen otros preceptos aplicables a ella que los referentes a su licitud y publicidad, y a su organización externa en relación con la intervención gubernativa; pero no reguló — porque no le correspondía- el régimen jurídico que exige la realidad económica del fenómeno.

La especialidad de la naturaleza cooperativa tampoco encuentra cobija en el Código Civil español hecho extensivo a Cuba en 1889, pues resultaba contraria al ánimo de lucro previsto como elemento constitutivo de las sociedades tipificadas en este cuerpo jurídico.

Sobre esta base es posible afirmar que la legislación colonial que rigió la cooperativa en la Cuba española fue reflejo de su tiempo, en tanto mostró el escepticismo y las incomprensiones del legislador acerca de un fenómeno cuya identidad jurídica aún no se perfilaba. Resultado de ello, no encontró la institución amparo legal apropiado para desarrollar su doble aspecto económico y social.

\section{La Constitución de 1940}

En tránsito hacia una nueva regulación jurídica de la cooperativa, pero aún bajo la vigencia e influencia de la legislación colonial, en la Cuba republicana se dictaron varias normas tocantes de la cooperativa y generalmente administrativas, destinadas a regular las ayudas económicas, su uso, disfrute y limites; arrendamientos colectivos para constituirlas de explotación agrícola o pecuaria; reglamentaciones laborales; régimen de inscripción; creación, control e inspección; etc. ${ }^{7}$. Estas normas carecieron de la especialización, la profundidad, la generalidad y la coherencia necesarias para impulsar la institución objeto de estudio de forma sistematizada e integral, en función de la satisfacción de las múltiples necesidades económicas y sociales de la población ${ }^{8}$; sino que más bien fue la oligarquía quien la utilizó como fachada para hacer valer sus intereses ${ }^{9}$.

Con la Constitución de 1940, influida por lo más avanzado del constitucionalismo social del momento, por primera vez se supra-orde-

7 V. gr.: Ley de 2 de septiembre de 1937; Decreto No. 85 de 1938; Decreto No. 2687 de 1940. Vid. BORGES, M. A.: Compilación ordenada y completa de la legislación cubana de 1899 a 1950 (ambos inclusive), Vol. II, Ed. Lex, La Habana, 1952, pp. 1315-1327; y Fernández Peiso, A.: La cooperativa. Bases para su legislación en Cuba, Ed. Ciencias Sociales, La Habana, 2012, p. 181 y ss.

8 En esta etapa, los sectores populares cubanos se apoyaron mucho más en el movimiento sindicalista para luchar por sus aspiraciones socioeconómicas, lo cual ha sido bien documentado en PICHARDO, H.: ob. cit, t. IV (Primera Parte), pp. 194 y ss.

9 Vid. Idem, t. III, p. 521. 
naron en el país rasgos suficientes para superar la normativa colonial extrapolada al territorio, por lo que puede identificarse a partir de este momento, una segunda etapa en el tracto histórico del régimen jurídico de la cooperativa.

Para justificarlo vale destacar primeramente su reconocimiento en el artículo 75, cuyo contenido resalta por lo adelantado de considerarla como empresa, teniendo en cuenta que la $\mathrm{ACl}$ lo hace por primera vez en 1995. Este criterio consigue distinguirla de las entidades caritativas o benéficas, con las que se mezclaba o confundía en la legislación ordinaria precedente.

Además, la ubicación de este precepto en la Sección Primera del Título Sexto, dedicada al «Trabajo», y no en la Sección Segunda destinada a la «Propiedad», la convierte en una alternativa laboral para la sociedad que necesitaba nuevas fuentes de empleo. Para Fernández PEISO, ello evidencia el criterio constituyente de que «...su naturaleza diferenciada deviene de su contenido sociológico y no de su contenido patrimonial, y que este contenido patrimonial está destinado a realizar una actividad de empresa con todas sus consecuencias, al servicio de sus miembros» ${ }^{10}$. Sin embargo, el precepto es omiso con respecto al contenido y el valor asociativo de la institución, reservando al legislador - que nunca las ejerció - plenas facultades para regular su definición, constitución y funcionamiento ${ }^{11}$; lo cual alcanza mayor importancia si se tiene en cuenta que las múltiples necesidades sociales a cuya satisfacción podía contribuir la cooperativa, superan el título de la sección constitucional en que se ubica («Trabajo»).

Estas carencias en el magno texto contrastan con criterios sobre la cooperativa presentes en la doctrina constitucional patria de estos años, donde se observaba un claro conocimiento sobre la naturaleza de la institución y la obra teórica que la sustenta. En tal sentido destaca Álvarez Tabío, quienes después de referenciar a Gide, Fourier y otros, concluye que «la cooperativa es una especie de asociación cuyas finalidades son: eliminar el intermediario, conjugar los intereses, satisfacer las necesidades sociales y mejorar las condiciones económicas de sus miembros..., es la única institución capaz de eliminar el antagonismo entre el individualismo y el socialismo, constituye una ley natural pode-

10 Ibidem, p. 60.

11 La única exigencia del constituyente hacia el legislador ordinario fue la de evitar que sirvan las cooperativas para eludir o adulterar las disposiciones que para el régimen del trabajo establece la propia Constitución [Vid. artículo 75 de la Constitución de la República de Cuba de 1940. Gaceta Oficial de la República de Cuba No. 464, de 8 de julio de 1940]. 
rosa que ha de marcar el futuro de las sociedades..., su desarrollo, en suma, conduce a la eliminación del capitalismo.... ${ }^{12}$.

Por otra parte, pese a que la Constitución no establece pautas concretas para asegurar la autonomía e independencia de la cooperativa frente a la Administración o el capital privado, resultó positivo que en el artículo 213, inciso c), emitiera un mandato a los gobiernos municipales para promoverlas en sus formas de producción y de consumo, a la vez que reconoció su vocación para el servicio público ${ }^{13}$.

Estos aspectos positivos fueron resultado de avanzados criterios sobre la institución que trascendieron desde los debates constituyentes. Entre otros destacó FERnÁndez de CASTRO, quien ponderó a las cooperativas como alternativa válida ante la ineptitud de las instituciones públicas, inclinándose hacia la «...organización de cooperativas porque los particulares construyen y administran mejor y más barato que los organismos oficiales, y el ciudadano que aspira a su mejoramiento económico y cultural, debe realizar algún esfuerzo y nunca esperarlo todo de la dádiva oficial, que desmoraliza su dignidad y condición de hombre libre» ${ }^{14}$. Más adelante ofrecía experiencias propias como argumentos.

En el mismo sentido el delegado ReY también reafirmó tal criterio, al considerar a las cooperativas «... una idea brillante, porque se ha demostrado que esas iniciativas (...) bajo la dirección y organización de elementos que conocen las necesidades de sus zonas, que al cabo son las de ellos mismos, darán frutos efectivos y servicios eficaces (...), y mucho de eso que debería hacer el gobierno ahora, y que no hace, podrá ser logrado por la iniciativa fecunda de esas cooperativas, si efectivamente nosotros ponemos en sus manos medios económicos bastantes... $\rangle^{15}$.

Lamentablemente, estaba la institución inserta en un contexto histórico-político que le impidió germinar con la suficiente fuerza como para ser más que un medio de sobrevivencia de reducidos sectores marginados económicamente o un mecanismo empleado por algunos grupos influyentes como fachada para enriquecerse de las

12 Álvarez Tabío, F.: Teoría General de la Constitución Cubana, Ed. Jesús Montero, La Habana, 1946, pp. 148 y 149.

13 «Artículo 213. Corresponde especialmente al Gobierno Municipal: c) ...propender al establecimiento de cooperativas de producción y de consumo..., todo con carácter de servicio público».

14 Acta de la sesión de debate 69, del 4 de junio de 1940, en LezCano y Mazón, A.M.: Constitución de Cuba (con los debates sobre su articulado y transitorias, en la Convención Constituyente), t. II, Ed. Cultural, La Habana, 1941, p. 736.

15 ldem. 
prebendas de los gobiernos mediante prácticas $\operatorname{corruptas}^{16} \mathrm{O}$, al menos, lucrativas ${ }^{17}$.

Pese a las carencias del régimen político-jurídico establecido por la Constitución de 1940 para el aprovechamiento de los rasgos inclusivos y democratizadores de la cooperativa, es criterio del autor de este trabajo que su tutela constitucional significó, en sí misma, una evolución en la regulación jurídica de la institución, lo cual se refuerza con el reconocimiento de su carácter de empresa, viable en sus diversos tipos y para la satisfacción de diversas necesidades socioeconómicas de la población, así como en la orden para su fomento desde lo local como mecanismo para favorecer servicios públicos.

\section{La legislación socialista}

El triunfo de la Revolución cubana en 1959 fue la antesala de una nueva etapa del régimen jurídico de la cooperativa. Pese a que la Ley Fundamental de este año mantuvo intactos los preceptos que referían a la cooperativa en el texto constitucional de 1940, así como a las carencias en el marco legal cooperativo complementario a la Ley Fundamental, prolongadas de las etapas anteriores, en los primeros meses proliferaron incipientes formas de cooperativas de diferentes tipos (trabajo y consumo) y en diferentes esferas de la economía ( $V$. gr.: agrícolas, ganaderas, carboneras, pesqueras, de producción de hilado, de maestros, etc.), que sirvieron como alternativa a los sectores más des-

16 Vid. Fernández Peiso, A.: La cooperativa. Bases para su regulación en Cuba, Ed. Ciencias Sociales, La Habana, 2012, p. 183. Un criterio diferente para explicar la poca eficacia de las aspiraciones constituyentes con la cooperativa ofrece Álvarez TABío: «Entre nosotros, el movimiento es muy débil debido, quizá, a la carencia de virtudes sociales de que se acusa a los latinos. Con razón se ha dicho que carecemos de disciplina y cohesión, y sin embargo nos sobra audacia e inteligencia crítica; que somos indisciplinados, poco previsores, de un espíritu individualista, rayano en el anarquismo, y que difícilmente nos sometemos voluntariamente a las decisiones de la mayoría. A todo esto hay que añadir que carecemos del espíritu de empresa, prefiriendo inmovilizar los capitales en inversiones seguras al emprender cualquier comercio o industria» [Álvarez TABío, F.: Teoría General de..., p. 149]. A opinión del autor de este trabajo, este es un criterio - cuando menos- subjetivo.

17 JIMÉNEZ SOLER referencia para 1958 cinco cooperativas entre las empresas más importantes del país (Cooperativa Avícola Industrial S.A; Cooperativa Azucarera Estrada Palma S.A.; Cooperativa de Cebadores de Camagüey S.A.; Cooperativa de Ómnibus Aliados S.A.; Cooperativa de Seguros Aliados del Transporte S.A.), todas ellas constituidas bajo la forma jurídica de sociedades anónimas [Vid. Jiménez SOler, G.: Las empresas de Cuba 1958, Ed. Ciencias Sociales, La Habana, 2004, pp. 219-222]. 
favorecidos del país para enfrentar las carencias económicas y sociales del momento ${ }^{18}$.

La Ley de Reforma Agraria de 17 de mayo de 1959, además de entregar la tierra a los campesinos, promovió en ellos el compromiso para cooperativizarse ${ }^{19}$, de lo cual se derivó un proceso asociacionista que tuvo un importante punto de referencia entrada la década de los 60 con la creación de las Cooperativas de Créditos y Servicios (CCS).

Debe resaltarse que en estos años, la cooperativa - generalmente- no gozó de autonomía ${ }^{20}$, ni fue resultado de la iniciativa

18 Vid. Fernández Peiso, A: "Notas características del marco legal del ambiente cooperativo cubano», en PIÑEIRO HARNECKER, C. (compiladora): Cooperativas y Socialismo. Una mirada desde Cuba, Ed. Caminos, La Habana, 2011, pp. 368 y ss. Además, existen referencias legales sobre la creación del Departamento de asociaciones y cooperativas de consumo y producción agrícola, comerciales e industriales del Ejército Rebelde, adscripto al Ministerio de Defensa [Vid. Artículo 1, apartado 5 de la Ley No. 100 de 23 de febrero de 1959, sobre "Creación de Departamentos de Asistencia Educacional y Técnica a las Fuerzas del Ejército Rebelde», Gaceta Oficial No. 33 de 26 de febrero de 1959]. No obstante, resulta sugerente que solo algunos meses después, con la extinción del Ministerio de Defensa, este Departamento quedó adscripto al INRA [Vid. Artículo 2 de la Ley No. 599 de 16 de octubre de 1959, sobre "Supresión del Ministerio de Defensa y adscripción de determinados Departamentos a los Órganos que se indican», Gaceta Oficial de 17 de octubre de 1959].

19 El artículo 43 de la Ley de Reforma Agraria de 17 de Mayo de 1959 [Gaceta Oficial de 3 de junio, reproducida en NAVARRETE ACEVEDo, C.R.: Legislación y documentos sobre Derecho Agrario cubano, Facultad de Derecho, Universidad de la Habana, La Habana, 1984, pp. 120 y ss.], ordenaba que "Siempre que sea posible, el Instituto Nacional de Reforma Agraria fomentará cooperativas agrarias...». A propósito de la aplicación de la Ley en este sentido se explicó: «En la formación de esas Cooperativas es donde tendrá el Gobierno las mayores dificultades a vencer. No será ya la lucha natural con los perjudicados..., sino la batalla con los beneficiados..., cuyo afán de mejorar y recibir las mayores ventajas los puede llevar a fricciones antagónicas que traigan el fracaso del plan. Es aquí donde los millares de campesinos y personas que reciban un bien tan grande, tienen que demostrar que son agradecidos; que aprecian lo que por ellos se ha hecho...» [López CASTILLO, R.: La Ley de Reforma Agraria, con explicaciones prácticas y formulaciones de escritos y acta notarial, Ed. Lex, La Habana, 1959, p. 51]. "Los campesinos se irán incorporando a la cooperativa en forma progresiva, según se vayan convenciendo con la prédica paciente y con el ejemplo concreto... y por esta vía irán al socialismo...» [AGUIRRE, S.: La Revolución Agraria, La Habana, 1961, p. 20].

20 La Ley de Reforma Agraria de 17 de mayo de 1959 [ob. cit.], en su artículo 43 establecía que "Las cooperativas agrarias que organice el INRA en las tierras que se disponga en virtud de lo preceptuado en esta Ley, estarán bajo su dirección, reservándose el derecho de designar los administradores de las mismas al objeto de asegurar su mejor desenvolvimiento en la etapa inicial de este tipo de organizaciones económicas y sociales y hasta tanto se le conceda por Ley una autonomía mayor»; y en el artículo 44 rezaba la propia Ley que «El INRA prestará su apoyo a las cooperativas formadas por 
consciente y espontánea de sus miembros, sino producto del accionar político del Gobierno Revolucionario que, con respaldo popular ${ }^{21}$, utilizó esta forma asociativa como instrumento para colectivizar beneficios sociales ${ }^{22}$; para promover la participación en la gestión de los recursos nacionalizados ${ }^{23}$; para asegurar las inversiones estatales 24 ; para optimi-

campesinos o trabajadores agrícolas..., según el régimen interno reglamentado por el propio Instituto». Tales facultades se materializaron, por ejemplo, en la Resolución No. 71 de 22 de octubre de 1959 [Vid. Gaceta Oficial de 30 de octubre de 1959], mediante la cual se creó la "Cooperativa de Obreros Henequeneros». Otro ejemplo lo constituye la Resolución No. 462 del INRA, de 6 de enero de 1964, que en su apartado segundo dispuso que «Se crea en cada provincia, bajo la dependencia del Delegado Provincial del Vice-Ministerio de la Producción Privada y Cooperativa, una empresa denominada Empresa Provincial de Servicios y Abastecimiento al Sector Privado..., teniendo a su cargo la prestación de abastecimiento, maquinaria y servicios técnicos a la producción privada» [Gaceta Oficial No. 8, de 14 de enero de 1964].

21 «Yo le pregunto al pueblo si está de acuerdo o no con que el Gobierno Revolucionario organice cooperativas de consumo en el campo para evitar que los campesinos paguen el doble por las mercancías (Exclamaciones de Aprobación)... Yo le pregunto al pueblo si está de acuerdo o no con que... los campesinos y carboneros... tengan sus cooperativas y sus camiones, para vender su carbón y no ser víctimas de la explotación (Exclamaciones de Aprobación)...» [CASTRo Ruz, F.: Discurso pronunciado ante el pueblo congregado en el Palacio Presidencial para reafirmar su apoyo al Gobierno Revolucionario y como protesta contra la agresión perpetrada contra el pueblo de La Habana por aviones extranjeros, el 26 de octubre de 1959].

22 «Nosotros no solamente estamos desarrollando los cultivos, sino que estamos preparando las condiciones de vivienda ideal, de escuela ideal, de asistencia ideal a sus necesidades de salubridad; en fin, que cada cooperativa será un modelo» [CASTRO RUz, F.: Discurso pronunciado en el acto de clausura del Primer Foro Nacional de la Reforma Agraria, efectuado en el Capitolio Nacional el 12 de julio de 1959].

23 «Así que nosotros, si expropiamos una colonia de caña de 100 caballerías, no la destruimos, reunimos a las familias de los obreros agrícolas de aquella finca, las llamamos, hacemos una cooperativa, fundamos un pueblo con escuelas, con campos deportivos, con dispensario médico, con tiendas del pueblo allí para que compren barato; se cultiva la finca en cooperativa con arados, se abona, se riega; producimos en 50 o 60 caballerías lo mismo que antes se producía en 100 ... Así que en vez de disminuir la producción lo que hacemos es aumentarla; en vez de dividir la colonia, la organizamos en una cooperativa, tiene todo el mundo trabajo, tiene todo el mundo crédito, se utiliza la tierra, se produce el doble y los campesinos reciben las utilidades» [CASTRO Ruz, F.: Discurso pronunciado en la ciudad de Santa Clara, el 21 de junio de 1959].

24 «En la primera etapa de las cooperativas, donde los campesinos van a recibir allí el producto íntegro de su trabajo, el Instituto Nacional de la Reforma Agraria solo interviene en la designación de los administradores, sencillamente para garantizar la producción... nosotros garantizamos la inversión correcta de todos los recursos de que se disponga» [CASTRO Ruz, F.: Discurso pronunciado en el acto de clausura del Primer Foro Nacional de la Reforma Agraria, efectuado en el Capitolio Nacional, el 12 de julio de 1959]. 
zar el aprovechamiento de los recursos ${ }^{25}$; y para ordenar y planificar la producción ${ }^{26}$.

Por otra parte, la posición del Ché durante los primeros años de la Revolución no favoreció la proliferación de las cooperativas. El líder revolucionario optó por implementar la organización económica del país como una gran maquinaria administrativa ${ }^{27} \mathrm{y}$, una vez fue nombrado Ministro de la Industria, creó un sistema de trabajo dentro del ramo destinado a fortalecer la gestión obrera de los medios de producción desde la empresa estatal28. Esta postura estuvo influenciada por las experiencias soviéticas de los koljoses que por estos años funcionaban - de facto- como formas de producción capitalistas ${ }^{29}$, generadoras de una superestructura que imponía un retorno progresivo al capitalismo ${ }^{30}$.

25 «Si tiene 200 caballerías, no se reparte; se hace una cooperativa, porque es mejor, porque usted utiliza el mismo regadío para todos, los mismos equipos para todos, la misma siembra para todos y entonces todo le sale más barato y a ese campesino, si en vez de darle un pedazo para que la cultive por su cuenta, la cultivan entre todos, ganan más y van a vivir en familia... Ahora van a ganar mucho más que antes, porque van a ganar el sueldo, más las utilidades de la venta... » [CASTRO RUz, F.: Discurso pronunciado en la ciudad de Santa Clara, el 21 de junio de 1959].

26 «Para mantener el consumo, para mantener la riqueza, para hacer la Reforma Agraria, no es posible repartir la tierra en un millón de pedacitos... Deben instalarse cooperativas en los lugares que sean propicios a este tipo de producción y hacerse un cultivo planificado de los terrenos» [CASTRO Ruz, F.: Discurso de clausura del Primer Congreso Provincial Campesino en Santiago de Cuba el 24 de febrero de 1959].

27 «No se entiende cómo pueden suprimirse los métodos coercitivos y remplazarlos por económicos. Si se hacen automáticos estos, se vuelve a una sociedad anárquica, si se guían por un plan central el Estado debe estar allí para velar. Lo que sucede o debe suceder es el cambio cualitativo siguiente: los obreros, el pueblo en general, decidirán sobre los grandes problemas del país..., en las localidades decidirán sobre problemas concretos..., pero el plan y la producción será obra de los especialistas, y no puede cambiarse por voluntades individualizadas, aunque sean en forma colectiva. El quid está en considerar la organización económica como una gran maquinaria; el pueblo tiene derecho a dictarle las normas a esa maquinaria, y vigilar que las cumpla, pero no introducirse en su engranaje» [Guevara de LA Serna, E. (Ché): Apuntes críticos a la economía política, II Edición, Centro de Estudios Ché Guevara, Ocean Press y Ed. Ciencias Sociales, La Habana, 2012, pp. 183 y 184].

28 Vid. «Selección de Actas de Reuniones efectuadas en el Ministerio de Industrias», en Guevara de la Serna, E. (Ché): ob. cit, Anexo 2, pp. 231 y ss.

29 Además de que se promovía la productividad en los koljoses con mecanismos económicos que alimentaban el ánimo de lucro en sus asociados, también denuncia el Ché casos referidos por la prensa soviética de koljoses que, para conseguir algunas de sus cosechas, dependían de la fuerza de trabajo que contrataban. Vid. GueVARA DE LA SERNA, E. (Ché): ob. cit, pp. 54 y 55.

30 Vid. Idem, pp. 27 y 187. 
A criterio del autor de este trabajo, precisamente porque no es la forma koljosiana la propia del socialismo, sino de la URSS31, no deben absolutizarse los criterios de que la cooperativa «...no es una forma socialista»32, o que «...tiene contradicciones frente a la gran colectividad...»"33, o que "...genera capitalismo y burguesía constantemente... $\rangle^{34}$, porque no son estos rasgos inherentes a la institución cuando se concibe y se manifiesta de acuerdo a su naturaleza social y no lucrativa; como tampoco lo son de la empresa estatal, aun cuando en ocasiones haya generado falta de sentido de pertenencia, ineficiencia, burocratismo y corrupción. En la orientación correcta de ambas «formas» (cooperativa y estatal), juegan un papel trascendental elementos de la superestructura como la cultura, la educación, la ética y el Derecho.

No obstante, la tendencia en la conformación de empresas en el país, tras la búsqueda de mecanismos de socialización de la propiedad, como vía para excluir «...la división de los hombres en poseedores de los medios de producción y desposeídos de ellos, y que las relaciones personales se basen en la colaboración y ayuda mutua» ${ }^{35}$, rápidamente desembocó en la estatalización de la propiedad y de su gestión a gran escala. Por ello, para 1968 prácticamente todos los medios de producción habían pasado a propiedad estatal, entendiéndose así terminado el proceso de socialización de la propiedad ${ }^{36}$.

De esta forma, se concentró la cooperativa en algunas fracciones del sector agropecuario de la economía. Para explicarlo, AzcuY razona — con base en el pensamiento de Fidel CASTRO Ruz - que "...hubiera sido un retroceso desde el punto de vista social convertir a los obreros en propietarios cooperativos. La organización cooperativa quedó entonces como un escalón de avance para los campesinos que continuaban la explotación individual de la tierra» ${ }^{37}$. Es por ello que se promueve desde el poder, llegados los años 70, «....bajo la óptica de aproximarlas a las formas estatales de gestión existentes» ${ }^{38}$, la forma-

31 Vid. Ibidem, p. 166.

32 Ibidem, p. 119.

33 Ibidem, p. 104.

34 Ibidem, pp. 57 y 58.

35 Álvarez Tabío, F.: Comentarios a la Constitución socialista cubana, Ed. Pueblo y Educación, La Habana, 1989, p. 84.

36 Vid. Casullera Arrate, R.: «La Propiedad Personal en nuestra Constitución», en Revista Jurídica No. 10, La Habana, enero-marzo de 1986, pp. 50 y ss.

37 Azcuy, H.: "Análisis de la Constitución cubana», en Revista Papeles de la FIM No. 14, Madrid, 2000, p. 53.

38 Fernández Peiso, A: «Notas características del....», ob. cit., p. 369. Y es que la propiedad cooperativa fue entendida como «... una forma inferior de propiedad socialista, 
ción de las Cooperativas de Producción Agropecuaria (CPA). Estas nuevas formas cooperativas, significaron un progreso en el propósito político de socializar la propiedad ${ }^{39}$, pues con ellas los campesinos venden sus medios a la cooperativa y pasan a ser propietarios y trabajadores colectivos; a diferencia de las CCS que ya existían, donde la asociación es básicamente a efectos de obtener créditos y servicios del Estado, pero manteniendo sus miembros la condición de propietarios individuales de la tierra y demás medios de producción.

Estas dos únicas formas de cooperativización - campesinas- que efectivamente se consolidaron en el país (CCS y CPA), adquieren por fin reconocimiento legal tras la promulgación de la Constitución socialista cubana el 24 de febrero de 1976, en cuyo artículo 20 se tuteló el derecho de los agricultores pequeños a agruparse a los efectos de la producción agropecuaria y de obtener créditos y servicios estatales; derecho no reconocido a otros sectores del pueblo trabajador que para entonces no resultó necesario pues el Estado se erigía como garante de sus necesidades.

En cuanto a la naturaleza jurídica de la institución, en este precepto se entiende como forma de propiedad, criterio que sigue el Código Civil de 1987 al ubicarla en su TíTULO II: DERECHO DE PROPIEDAD, CA-

cuyo origen se debe a que en el socialismo todo el poder pertenece a los trabajadores, y el Estado es el propietario de todos los medios fundamentales de producción. Visto así la propiedad cooperativa es también propiedad social, pero con rango inferior a la estatal, ya que mientras esta forma de propiedad pertenece a toda la nación, la propiedad cooperativa pertenece al grupo de cooperativistas que se han integrado voluntariamente» [CANTÓN BLANCO, L.E.: CANTÓN BLANCO, L.E.: Conferencias de Propiedad y Derechos Reales, Ed. ENSPES, La Habana, 1982, p. 180]. De igual forma plantea el profesor MIR PÉREz que "La Revolución Socialista proclama la propiedad socialista, cuyo titular es el Pueblo, con la forma de Propiedad Estatal Socialista; la cooperativa agropecuaria de los agricultores pequeños, forma secundaria socialista...» [MIR PÉREZ, J.: Derecho de Propiedad, t. I, Facultad de Derecho, Universidad de Oriente, ENSPES, 1984, p. 3].

39 En el Primer Congreso del Partido Comunista de Cuba pudo apreciarse la voluntad política de propiciar la colectivización de los campesinos y obreros agrícolas cuando en el punto Sexto de la Resolución Sobre la Cuestión Agraria y las Relaciones con el Campesinado, se deja en claro que "Se trata de un proceso gradual, cuyo ritmo más o menos rápido dependerá de dos factores que ejercen entre sí una influencia recíproca: el desarrollo de las fuerzas productivas y la profundización de la conciencia del campesinado y del trabajador. Como ambos factores están constantemente avanzando en nuestro país, este proceso puede ser de mayor o menor moderación, pero, desde luego, no será eterno». De igual forma, en el punto Décimo se ordena que «Bajo la orientación y guía del Partido, corresponde a la ANAP un papel fundamental en la paciente y sistemática labor de divulgar, esclarecer, convencer, conquistar, a cada familia campesina para el propósito de marchar, llegado el momento, hacia formas socialistas de producción...». 
PÍTULO II: FORMAS DE PROPIEDAD; SECCIÓN TERCERA: PROPIEDAD COOPERATIVA. Esta concepción, igualmente presente en la legislación especial agraria (Ley No. 36, De Cooperativas Agropecuarias, de 22 de julio de 1982 y con posterioridad la vigente Ley No. 95, De Cooperativas de Producción Agropecuaria y de Créditos y Servicios, de 2 de noviembre de 2002 que derogó la anterior), lleva a advertir que «...el modelo jurídico adoptado, está distanciado de la naturaleza social de la institución cooperativa, pues en él se privilegia el componente administrativo-patrimonial, sobre el asociativo.... 40 , lo cual ha obstaculizado que el Derecho consolide a la institución conforme a su identidad y que se aprovechen sus cualidades intrínsecas en función del desarrollo del país.

El criterio agrarista y patrimonialista ha caracterizado la concepción de la cooperativa en el diseño constitucional socialista cubano. El debate en torno a su definición en el seno de la Asamblea Nacional del Poder Popular (ANPP), con motivo de la reforma constitucional del 10 de julio de 1992 lo evidenció.

Existió consenso en que la preceptiva constitucional, al caracterizarlas, debía exaltar su importancia; por eso el diputado y entonces Presidente de los Consejos de Estado y de Ministros CASTRo Ruz (Fidel), insistió en «...lo de "avanzada y eficiente» porque hay que elogiarlas, moralmente hay que apoyar a las cooperativas ${ }^{41}$. Pero de igual forma fue aceptado sin discusión que se regulara constitucionalmente como una «forma de propiedad» 42 , algo que en sí mismo no es negativo porque resguarda la titularidad sobre sus bienes; pero que al entenderse como su naturaleza jurídica, reduce sustancialmente sus múltiples potencialidades asociativas.

Importante polémica generó el hecho de que con el Proyecto de Reforma se pretendiese conservar la definición de la cooperativa como una «forma colectiva de propiedad», pues para algunos no indicaba con claridad su lugar y papel al interior del sistema socioeconómico establecido, algo que evidenció los prejuicios sobre su congruencia con el socialismo o, más bien, con el modelo de socialismo asumido.

40 Fernández Peiso, A.: Lecturas en pro del cooperativismo, ante las imprescindibles transformaciones económicas del socialismo cubano, Ed. Universo Sur, Cienfuegos, 2006, p. 27.

41 Acta contentiva de la discusión del Proyecto de Ley de Reforma Constitucional; Décimo Período Ordinario de Sesiones, Tercera Legislatura de la Asamblea Nacional del Poder Popular; días 10, 11 y 12 de julio de 1992, p. 185.

42 Idem. 
En este sentido, la diputada CASTRO AbRALDES expresó que: «... cuando se habla de propiedad social, existen diferentes formas. Cuando enmarcamos entre las formas de propiedad social la propiedad cooperativa, la identificamos como una propiedad colectiva. En la literatura económica (...) a este tipo de propiedad cooperativa nuestra le ponemos el apellido de propiedad colectiva socialista... [Por tanto] pienso que la terminología más adecuada para utilizar aquí constitucionalmente es la de «propiedad colectiva socialista»»"43.

Sobre esta misma cuestión ya había precisado el diputado CASTRO Ruz (Fidel), que no era suficiente con que se declarara a la cooperativa como «una forma avanzada y eficiente de propiedad colectiva», pues esta definición genérica también serviría a la sociedad anónima y no existía la intención de confundirlas ${ }^{44}$.

A estos criterios se opuso el diputado Rodríguez Rodríguez afirmando que «...la forma colectiva puede ser socialista o puede no ir inclinada al socialismo», a lo que agrega que la cooperativa "No puede ser "propiedad socialista» porque no siempre la forma colectiva va dirigida al socialismo», prefiriendo más bien el término de "propiedad colectiva» ${ }^{45}$. También se defendió por el diputado RAMírEz CRUz no incluir el adjetivo "socialista» para caracterizar a esta forma de propiedad, pues a su consideración «...el concepto de propiedad socialista es la propiedad de todo el pueblo y ésta es una propiedad de grupo, es decir, no es una propiedad de toda la sociedad» ${ }^{46}$.

Pero, en última instancia, el verdadero carácter de la propiedad no viene determinado por cómo se proclame ésta en la norma jurídica, sino por la naturaleza de las relaciones de producción en que se desenvuelva ${ }^{47}$. Más importantes que adjetivos para acompañar a la institución, es que el Derecho le perfile objetivos socialistas a los que habrá de contribuir de acuerdo a los límites que el propio Derecho establezca. Además, no existe razón para desconocer que se trata de una aspiración, que como cualquier otra debe ser conquistada.

En busca de consenso para este debate, el Presidente de la Asamblea propone sustituir la fórmula original de «forma de propiedad colectiva», no ya por la de "forma de propiedad colectiva socialista» propuesta, sino por la que definitivamente se aprobó: «forma de pro-

\footnotetext{
43 Ibidem, pp. 188-189.

44 Vid. Ibidem, p. 185.

45 Vid. Ibidem, p. 186.

46 Ibidem, p. 187.

47 AzCuY, H.: ob. cit., p. 52
} 
ducción socialista» ${ }^{48}$, lo cual introduce sobre lo regulado, a consideración del autor, una ambigüedad en su definición constitucional, lo que agudizó la falta de claridad sobre los rasgos y funciones que se le asignaban.

Sobre el carácter agropecuario de la cooperativa, las interpretaciones doctrinales al texto constitucional cubano de 1976 habían estado divididas: Para algunos «la Constitución reconoce a la cooperativa como una propiedad agraria, sin hacerla extensiva a ningún otro tipo de explotación económica» ${ }^{49}$, viéndose en su definición constitucional un límite para su expansión legal a otros sectores de la economía. Para otros en cambio, «la enumeración constitucional de las formas de propiedad no es taxativa, por lo cual es factible que la ley establezca la autorización para otras modalidades o posibilidades de la propiedad cooperativa» ${ }^{50}$.

Al respecto, durante el proceso de discusión y aprobación del Proyecto de Reforma Constitucional de 1992, el diputado CASTRO Ruz (Fidel) alertó que «...toda la perestroika empezó hablando de cooperativas, de cooperativas de servicios, cooperativas de tiendas, cooperativas de negocio, y todo camino del capitalismo a toda velocidad. No debemos olvidarnos de esos otros pequeños riesgos de que parezca exaltada demasiado la propiedad cooperativa. Creo que debiéramos distinguir un poco y decir "de propiedad cooperativa agrícola», para distinguirla frente a cualquier tendencia de querer generalizar el concepto de la cooperativa, porque si funciona en la agricultura, debemos decir «forma de propiedad cooperativa agrícola»» ${ }^{51}$.

Justificada era la prudencia, pues pervivía el fantasma del derrumbe del campo socialista y resonaban los retóricos augurios que, desde afuera del país, aseguraban que el régimen socioeconómico y político cubano se revertiría. Este entramado de circunstancias negativas, creaba una coyuntura compleja para implementar — con las inevitables dosis de experimentación - los cambios que el modelo económico requería.

48 Vid. Acta contentiva de la discusión del Proyecto de Ley de Reforma Constitucional; Décimo Período Ordinario de Sesiones, Tercera Legislatura de la Asamblea Nacional del Poder Popular; días 10, 11 y 12 de julio de 1992, p. 188.

49 AzCuY, H.: ob. cit., p. 54.

50 Vega Vega, J.: Derecho Constitucional revolucionario en Cuba, Ed. Ciencias Sociales, La Habana, 1988, p. 159.

51 Acta contentiva de la discusión del Proyecto de Ley de Reforma Constitucional, Décimo Período Ordinario de Sesiones, Tercera Legislatura de la Asamblea Nacional del Poder Popular, días 10, 11 y 12 de julio de 1992, pp. 189-190. 
Conclusión positiva de ese contexto fue la de no continuar imitando aquel modelo de socialismo, mucho menos en una etapa que, mostrada en un inicio como alternativas de solución a sus crónicas deficiencias, hubo de llevarlo definitivamente a fenecer ${ }^{52}$. Pero, no debe suponerse que algún elemento no sería de factible implementación en Cuba tan solo porque formó parte de la perestroika ${ }^{53}$. A criterio del autor de este trabajo, la expansión del sector cooperativo no es, en sí misma, un factor de riesgo para la continuidad del socialismo: cada circunstancia debe valorarse con sentido del momento histórico y ningún fenómeno puede juzgarse bien fuera de su contexto. «No hay otra alternativa posible, es necesario ser dialécticos y creadores» ${ }^{54}$, solo con respeto a esta premisa podrá medirse eficazmente la actual factibilidad de las cooperativas en sectores de la economía cubana diferentes al agropecuario.

En un balance sobre estos debates en la Asamblea Nacional del Poder Popular, destacan las tergiversaciones político-ideológicas existentes en torno a la institución, que afectaban su concepción teórica e impedían que se favoreciera en su definición constitucional. Estas tergiversaciones, impidieron que su régimen jurídico constitucional $-\mathrm{y}$ legal- facilitara su contribución a la satisfacción de las necesidades socioeconómicas de sus miembros y de la comunidad en que se desarrolla.

Posterior a la reforma y como resultado del contexto de crisis económica y de la reducida sostenibilidad del modelo agrario ampliamente

52 La perestroika o reestructuración de la economía soviética, desarrollada por Mijaíl Gorbachov a partir de 1985 en la URSS, en sus inicios (1985-1987) pretendió perfeccionar la gestión económica en los marcos del socialismo, pero la aplicación de la política diseñada con este fin desembocó (1988-1989) en una crisis que conlleva a que se comience a buscar un cambio fuera del sistema socialista, para finalmente (1990-1991) abandonar esta opción y asimilar la economía de mercado capitalista como única solución a los problemas económicos que enfrentaba aquel país. Vid. RodríGUez GarCíA, J.L.: «La perestroika en la economía soviética 1985-1991», en Revista Internacional Marx Ahora, No. 22, La Habana, 2006, pp. 57-67.

53 De hecho, no sería acertado culpar del descalabro a que conllevó la nueva política de reestructuración económica tan solo a sus precursores, debemos recordar que ella estuvo precedida de varias décadas plagadas de errores de todo tipo: el proceso de colectivización forzosa de los campesinos; el terror político desarrollado por Stalin; la ausencia de un auténtico centralismo democrático como método sistemático de gobierno, la separación cada vez mayor del Partido - y sobre todo su máxima dirección- de las masas populares; la burocratización del trabajo político ideológico; el formalismo; el oportunismo y el dogmatismo; son solo algunos de ellos. Vid. Ibidem, p. 53.

54 CAStro Ruz, F.: «Reflexión «Regalo de reyes»», La Habana, 14 de enero de 2008, recuperado de www.cubadebate.cu/reflexiones-fidel/2008/01/14/regalo-reyes, el 23 octubre de 2010. 
estatalizado, otro acontecimiento importante en el ámbito cooperativo ocurre cuando el Estado cubano se vio forzado a aprobar el Decreto Ley 142 del 20 de septiembre de 1993, "Sobre las Unidades Básicas de Producción Cooperativa» y a crear, por su iniciativa, estas nuevas formas de producción cooperada (UBPC) a partir del fraccionamiento estructural de la gran propiedad agrícola estatal. De esta forma, el Estado conservó la propiedad de la tierra y convirtió a los obreros agrícolas en usufructuarios de ella y propietarios del resto de los medios de producción necesarios para su explotación.

En las UBPC, es donde mejor se observa que al regularse las relaciones jurídicas entre el Estado y las cooperativas agropecuarias en Cuba, se asumió un modelo absorbente ${ }^{55}$ o de dependencia ${ }^{56}$ que ha limitado de forma sustancial la autonomía que debe caracterizarlas. Este planteamiento se basa - entre otros aspectos- en la intervención de la autoridad pública al determinarse su objeto social; en las limitaciones legales a la libertad contractual; en el carácter formal de las relaciones contractuales con las empresas estatales y en los requerimientos administrativos para su constitución y disolución ${ }^{57}$. Por tanto, la UBPC «es una forma de organización empresarial, fruto del rediseño de la propiedad estatal, que vincula en un sistema peculiar de relaciones económicas la gestión cooperativa con la dirección centralizada»58, pero no surgió como resultado de la iniciativa consciente de sus asociados, ni cuenta con todos los caracteres básicos de una cooperativa.

Sobre esta base, puede concluirse que la regulación jurídica de la cooperativa en el diseño socialista cubano ha respondido más al dirigismo de resortes políticos supra-ordenadores, legitimados por el noble propósito de socializar la propiedad sobre los medios de producción y/o los resultados de su producción, que a la consciente voluntad de los asociados de utilizar a la cooperativa como un medio para la satisfacción de

55 Vid. CRacogna, D.: «La legislación cooperativa en el mundo de hoy», documento presentado en el Seminario de Legislación Cooperativa en Uruguay el 22 de noviembre de 2001, recuperado de http://www.neticoop.org.uy/article118.html, el 12 de febrero de 2013.

56 Vid. Rosemburg, T.: La Empresa Cooperativa, Ed. CEAC, Barcelona, 1985, p. 104; referenciado por Fernández Peiso, A.: Lecturas en pro..., ob. cit., p. 137.

57 Vid. Rodríguez Musa, O.: «La autonomía cooperativa y su expresión jurídica. Una aproximación crítica a su actual implementación legal en Cuba», en Boletín de la Asociación Internacional de Derecho Cooperativo, No. 47, Universidad de Deusto, Bilbao, 2013, pp. 142 y ss.

58 LÓpez LABRADA, A. y Rodríguez MemBrado, E.: «La UBPC: forma de rediseñar la propiedad estatal con gestión cooperativa», en PINEEIRO HARNECKER, C. (compiladora): Cooperativismo y Socialismo..., ob. cit., p. 362. 
sus necesidades socioeconómicas comunes y la de su comunidad. Como consecuencia de ello, se ha constreñido a los agricultores pequeños, pese a ser Cuba un Estado de trabajadores, el derecho a asociarse en cooperativas, desaprovechándose las potencialidades de la institución fuera de esta esfera. Además, su naturaleza jurídica se redujo a forma de propiedad, obviándose la riqueza axiológica de las relaciones sociales que genera; se limitaron sus fines constitucionalmente reconocidos a la producción agropecuaria y la obtención de créditos y servicios estatales, cuando ella puede dirigirse a muchos otros propósitos socioeconómicos; y se le ha configurado un ambiente institucional con altos niveles de dependencia y paternalismo estatal, como si el Derecho no pudiese equilibrar, en pos del desarrollo sostenible del país, el ejercicio de la autonomía cooperativa y la responsabilidad social que le corresponde.

Estos elementos, a criterio del autor, permiten afirmar que el antiguo modelo agrarista del cooperativismo nacional se encuentra agotado y urgido de estudios teóricos con miradas que trasciendan sus estrechos márgenes, para ofrecerle perspectivas más generales e integrales.

\section{El actual proceso de expansión de las cooperativas en Cuba. Limitaciones y perspectivas jurídicas}

El VI Congreso del Partido Comunista de Cuba, celebrado en abril de 2011, aprobó los Lineamientos de la Política Económica y Social del Partido y la Revolución con el objetivo de sentar las pautas necesarias para conducir el proceso de actualización del modelo económico socialista en que hoy se encuentra inmerso el país. Los puntos del 25 al 29 de estos Lineamientos, agrupados bajo el título de "LAS COOPERATIVAS», establecieron los aspectos básicos para la inserción de éstas en un nuevo «MODELO DE GESTIÓN ECONÓMICA» que planeó expandir estas formas asociativas hacia otras esferas de la economía diferentes a la agropecuaria ${ }^{59}$.

59 Estos Lineamientos fueron revisados por el VII Congreso del Partido Comunista de Cuba en abril de 2016, y el nuevo documento ratifica y actualiza esta política. Vid. VII Congreso del PARtido Comunista de Cuba: Actualización de los Lineamientos de la Política Económica y Social del Partido y la Revolución para el período 2016-2021, abril de 2016, aprobados además por la Asamblea Nacional del Poder Popular en julio del propio año, recuperado de http://www.cubadebate.cu/especiales/2016/09/13/vea-el-textointegro-de-la-actualizacion-de-los-lineamientos-para-el-periodo-2016-2021-pdf/, el 2 de octubre de 2016, puntos 15 y 16 principalmente. 
Los referidos Lineamientos relativos a las cooperativas, fueron desarrollados originalmente por un paquete legislativo con carácter experimental. Estas disposiciones, entraron en vigor el 11 de diciembre de 2012 cuando se publicó la Gaceta Oficial Extraordinaria No. 53, contentiva del Decreto-Ley No. 305, de 15 de noviembre de 2012, «De las Cooperativas No Agropecuarias» y del Decreto No. 309 del 28 de noviembre de 2012, "Reglamento de las Cooperativas no Agropecuarias»; entre otras normativas que configuraron el marco regulatorio provisional para las nuevas Cooperativas no Agropecuarias (CNA) en Cuba.

Al acontecer estas novedades legislativas, que inmediatamente tuvieron una repercusión práctica ${ }^{60}$, se identificaron como limitaciones jurídicas para la cooperativa en Cuba, que responde a insuficiencias arrastradas de etapas precedentes y a otras más recientes, las siguientes $^{61}$ :

1. Insuficiencias en su regulación constitucional (artículo 20 de la Constitución cubana de 24 de febrero de 1976), en tanto el magno texto no tutela el derecho de otros trabajadores diferentes a los agricultores pequeños a asociarse en cooperativas $y$, por tanto, no reconoce su existencia más allá del sector agropecuario de la economía. Además, reduce su naturaleza jurídica a forma de propiedad, descuidando así el vínculo cooperativo, la finalidad de servicio que le asiste, y los valores y principios que le resultan consustanciales. En definitiva, no contiene una institucionalización de la cooperativa como figura autónoma, en relación de complementariedad con otros entes públicos y privados, para la satisfacción de las necesidades socioeconómicas de las personas.

2. Ausencia de una concepción armonizadora y homogénea acerca de las cooperativas y su naturaleza jurídica, lo cual está

60 Las cooperativas no agropecuarias aprobadas en el país fueron 514. Aproximadamente el $88 \%$ de estas nuevas cooperatvas se concentra en tres sectores: Comercio, Gastronomía, y Servicios Técnicos y Personales (59\%); Construcción (19\%); e Industria (10\%). Vid. Oficina NACIONAL DE EstAdístiCAS e INFORMACIÓN (ONEI): «Listado de cooperativas no agropecuarias con su código, marzo de 2016, recuperado de www. one.cu/ryc/cambian/CNoA.rar, el 4 de octubre de 2016. En este mismo sentido «Autorizadas 16 cooperativas no agropecuarias en primer trimestre de 2016», recuperado de http://www.cubadebate.cu/noticias/2016/06/29/autorizadas-16-cooperativas-noagropecuarias-en-primer-trimestre-de-2016/, el 4 de octubre de 2016.

61 Vid. Rodríguez Musa, O.: La constitucionalización de la cooperativa. Una propuesta para su redimensionamiento en Cuba. No. 1 Coletânea IBECOOP, Ed. Vincere Asociados, Brasilia-DF, 2017, p. 110. 
directamente ligado a la no existencia de una legislación general unificadora y armonizadora del sector, que se expresa actualmente en la pluralidad de normas, dispersas (algunas con carácter experimental), que lo fracturan entre lo agropecuario y lo no agropecuario, y que presentan poca sistematicidad y coherencia entre sí. Esto unido a antinomias o contradicciones derivadas del reglamentarismo excesivo y de la diversidad en las bases contextuales a las que responden. De esta forma quedan en entredicho los rasgos y principios de la institución, así como la posibilidad de determinar normas supletorias aplicables sin distorsionarle su naturaleza. Todo ello redunda en afectaciones a la identidad de las cooperativas y al empoderamiento del que precisan para transformar su medio económico-social.

3. Permanencia de un modelo absorbente en las relaciones de la cooperativa con el Estado, que aunque en los últimos años muestra una tendencia a su flexibilización ${ }^{62}$, afecta la autonomía de esta desde el proceso para su constitución y hasta el de su disolución, pasando por la determinación de su objeto social, por la planificación de su actividad económica y por las características de sus relaciones contractuales. A esto se une la ampliación de los entes públicos que interactúan con las cooperativas promoviéndolas, autorizándolas, calificándolas y controlándolas, los cuales se han diversificado tanto como las esferas de la economía en que estas funcionan y, con ellos, los métodos, políticas y disposiciones que se le aplican. Tal atomización limita la consolidación de la identidad de la figura por sobre la esfera de la economía en que se desarrolle.

En la agudización de los efectos negativos de estas limitaciones ha incidido la generalizada falta de cultura jurídico-cooperativa, que ha derivado en que el legislador, el aplicador de la norma cooperativa y la sociedad en general, arrastren hacia estas formas asociativas los esquemas de la empresa estatal o los importen de las formas capitalistas.

Como resultado de estas limitaciones en la plataforma jurídico-institucional, el proceso de expansión de las cooperativas hacia otras esferas de la economía nacional se ha visto afectado. Entre las dificultades reconocidas oficialmente está la apropiación indebida de recursos y de ingresos; las personas que fungen como socios de varias cooperativas a la vez; deficiencias en los registros contables; utilización de créditos bancarios con fines diferentes para los cuales se otorgaron; y algunos

62 Vid. Rodríguez Musa, O.: «La autonomía cooperativa... », ob. cit., pp. 142 y ss. 
hechos de corrupción. Además se ha dicho que algunas cooperativas han actuado como empresas privadas, donde el presidente ejerce como si fuera el dueño, con un mínimo de socios, a la vez que realizan su gestión fundamentalmente contratando los servicios de trabajadores por cuenta propia como asalariados, para luego repartir las utilidades solo entre los socios, poniéndose de manifiesto también diferencias significativas e injustificadas en los ingresos que reciben estos últimos ${ }^{63}$.

Sobre esta base, se ha decidido, "antes de continuar avanzando en la creación de nuevas cooperativas, consolidar lo avanzado, generalizar los aspectos positivos, que no son pocos, y enfrentar resueltamente las ilegalidades y otras desviaciones que se apartan de la política establecida» ${ }^{64}$. Esta directriz ha derivado en "hacer más efectivo el control y la fiscalización» ${ }^{65}$ desde el Estado sobre las cooperativas, así como en la decisión de extinguir algunas de las nuevas cooperativas que se habían autorizado66.

A tono con ello, en lo que va de año se han publicado nuevas normas jurídicas cooperativas, tanto para el sector agropecuario (Gaceta Oficial No. 37 Ordinaria de 24 de mayo de 2019, contentiva del Decreto-Ley No. 365 «De las Cooperativas Agropecuarias» y del Decreto No. 354 «Reglamento del Decreto-Ley de las Cooperativas Agropecuarias») como para el no agropecuario (Gaceta Oficial No. 63 Ordinaria de 30 de agosto de 2019, contentiva del Decreto-Ley No. 366 «De las

63 Vid. Vega Mato, Y. (Segunda Jefa del Área de Perfeccionamiento a Entidades de la Comisión Permanente para la Implementación y Desarrollo), en PUIG MENESES, Y.: «Autoridades explican nuevas medidas respecto a cooperativas no agropecuarias», La Habana, 9 de agosto de 2017, recuperado en http://www.cubadebate.cu/ noticias/2017/08/09/autoridades-explican-nuevas-medidas-respecto-a-cooperativas-noagropecuarias/, el 12 de septiembre de 2017.

64 CAStRo Ruz, R.: «Discurso pronunciado por el General de Ejército Raúl Castro Ruz, Primer Secretario del Comité Central del Partido Comunista de Cuba y Presidente de los Consejos de Estado y de Ministros, en la clausura del IX Período Ordinario de Sesiones de la VIII Legislatura de la Asamblea Nacional del Poder Popular», La Habana, 14 de julio de 2017, Versiones Taquigráficas del Consejo de Estado en Periódico Granma, 15 de julio de 2017.

65 Vega Mato, Y. (Segunda Jefa del Área de Perfeccionamiento a Entidades de la Comisión Permanente para la Implementación y Desarrollo), en PUIG MENESES, Y.: «Autoridades explican nuevas...», ob. cit.

66 Vid. CuBA Debate: «Cierran temporalmente el mercado mayorista El Trigal», La Habana, 12 mayo 2016, recuperado de http://www.cubadebate.cu/noticias/2016/05/12/cierran-el-mercado-mayorista-el-trigal/, el 12 de septiembre de 2017; y MINISTERIO DE FINANZAS Y PRECIOS: "Aprobada extinción de Cooperativa de servicios contables SCENIUS», La Habana, 7 agosto 2017, recuperado en http://www.cubadebate.cu/noticias/2017/08/07/ aprobada-extincion-de-cooperativa-de-servicios-contables-scenius/\#.Wb54j3uR7mM, el 12 de septiembre de 2017. 
Cooperativas no Agropecuarias» y del Decreto No. 356 «Reglamento de las Cooperativas no Agropecuarias»).

Sin embargo, a diferencia de lo que algunos esperábamos, las nuevas regulaciones no llegaron para unificar el sector, ni para establecer las bases generales y definitivas que pueden contribuir a su consolidación conforme a la identidad cooperativa universalmente reconocida. Por el contrario, estas normas no emanaron de la Asamblea Nacional del Poder Popular, pese a que en algunos casos derogan otras que sí poseen esta jerarquía67; conservan la división entre cooperativas agropecuarias y no agropecuarias; otorgan importantes facultades a instituciones del Estado que en breve desaparecerán con la implementación de la nueva Constitución de la República de Cuba de 2019 (V.gr.: Consejos de Administración Provincial68); y, en el caso del Decreto-Ley 366/2019 y su Reglamento, dirigidos a las CNA, no superan el carácter experimental de las regulaciones que derogan ${ }^{69}$, pese a los más de seis años trascurridos en este estado de inseguridad jurídica.

Pero, el cambio normativo más significativo en torno a las cooperativas cubanas llegó con el Magno Texto del 10 de abril de 2019, tras un proceso popular ampliamente participativo. En este sentido, es importante resaltar ante todo que la nueva Constitución, en su artículo 22 -inciso d) - reconoce la propiedad «privada: la que se ejerce sobre determinados medios de producción por personas naturales o jurídicas cubanas o extranjeras...». Llega así el oportuno fundamento para autorizar la creación de empresas privadas bajo formas jurídicas de naturaleza lucrativa. De esta forma, desaparecería la necesidad de aquellos que han usado a las cooperativas para encubrir este tipo de actividad económica.

Además, el propio artículo 22 de la nueva Carta Magna, en su inciso b), reconoce a la «propiedad cooperativa», como «la sustentada en el trabajo colectivo de sus socios propietarios y en el ejercicio efectivo de los principios del cooperativismo». La letra de este precepto po-

67 El Decreto-Ley 365/2019 deroga, entre otras disposiciones, a la Ley No. 95 «Ley de Cooperativas de Producción Agropecuaria y de Créditos y Servicios». Gaceta Oficial No. 72 Ordinaria de 29 de noviembre de 2002.

68 Los Consejos de Administración Provincial, facultados por el Decreto-Ley 366/2019 a presentan a la Comisión Permanente para la Implementación y Desarrollo, las solicitudes de creación de cooperativas (artículo 14, apartado 1), así como a autorizar la constitución de estas (artículo 13, inciso b), desaparecerán con la implementación de la Constitución de la República de Cuba recién aprobaba (Vid. artículos 170 al 184 de la Constitución de la República de Cuba. Gaceta Oficial No. 5 Extraordinaria de 10 de abril de 2019).

69 Vid. artículo 1 del Decreto-Ley 366/2019. 
see algunos aspectos que significan una evolución respecto a la vieja Constitución de 1976, así como otros elementos que generan incertidumbre y varias ausencias que podrían redundar en inercia, a saber:

- Evolución: Desaparece la perspectiva agrarista de la vieja Constitución. Ahora las cooperativas, sin importar el sector de la economía donde se desarrollen, tendrán protección constitucional. Además, se reconoce la pertinencia de unos «principios» que deben marcar el funcionamiento de estas instituciones, en tanto forman parte de un movimiento que las supera y las fortalece.

- Incertidumbre: Sin embargo, cabe preguntarse a qué «principios del cooperativismo» se refiere ahora el Constituyente, pues en Cuba nunca se han mencionado los enarbolados por la Alianza Cooperativa Internacional, y tampoco se ha utilizado un criterio uniforme para definirlos. Por tanto, diversas podrán ser las interpretaciones de la Constitución que —en este caso- realice el legislador.

- Inercia: En otro sentido, persiste la reducción de su naturaleza jurídica a «forma de propiedad», descuidándose el vínculo asociativo que implica, la finalidad de servicio que le corresponde, los valores que le resultan consustanciales y el ambiente institucional en el que - conforme a su identidad - se debe articular. Además, la formulación enfática respecto al «trabajo colectivo de sus socios propietarios» como sustento para las cooperativas, podría figurar como una limitación para constituir otro tipo de cooperativas diferentes a las de trabajo, como las de consumo o crédito (inexistentes hasta ahora en el país), que también se inspiran en necesidades socioeconómicas de carácter popular.

No obstante, ante estas dificultades, la voluntad del legislador y/o de la autoridad de aplicación, mucho podrían potenciar la realidad de las cooperativas.

\section{Conclusiones}

En Cuba, la regulación jurídica de la cooperativa históricamente no ha sido coherente con su identidad, en tanto se ha forzado su tipificación en otras figuras que no abarcan su doble contenido económico y social, o se le ha definido a partir de concepciones reduccionistas que no han favorecido su desarrollo acorde con la satisfacción de las necesidades populares. 
Al amparo del texto constitucional socialista de 1976 se potenció en el país un modelo agrarista, patrimonialista y con relaciones absorbentes hacia el Estado, que obstaculizó el óptimo aprovechamiento de la figura para la satisfacción de las necesidades socioeconómicas del pueblo.

Pese a ello, en la actualidad la cooperativa se está expandiendo hacia otras esferas de la economía nacional, sobre la base de un marco legislativo de carácter experimental. En este proceso, se han presentado dificultades que han desvirtuado el carácter asociativo de la institución y su finalidad de servicio a sus miembros y a la comunidad.

La nueva Constitución cubana de 2019, pese a la inercia de reducir la naturaleza jurídica de la cooperativa a "forma de propiedad» y a la parquedad que manifiesta respecto a los fines de la institución y a los principios que deben guiar su funcionamiento, abre una puerta al legislador para institucionalizar un movimiento socioeconómico que supere las limitaciones que hasta ahora presenta.

\section{Bibliografía}

1. Aguirre, S.: La Revolución Agraria, La Habana, 1961.

2. Álvarez Tabío, F.: Teoría General de la Constitución Cubana, Ed. Jesús Montero, La Habana, 1946.

3. Álvarez Tabío, F.: Comentarios a la Constitución socialista cubana, Ed. Pueblo y Educación, La Habana, 1989.

4. Azcuy, H.: "Análisis de la Constitución cubana», en Revista Papeles de la FIM No. 14, Madrid, 2000, pp. 11-144.

5. Borges, M.A.: Compilación ordenada y completa de la legislación cubana de 1899 a 1950 (ambos inclusive), Vol. II, Ed. Lex, La Habana, 1952, pp. 1315-1327.

6. Castro Ruz, F.: La Historia me Absolverá, edición anotada por Álvarez TABío, P. y Alonso Fiel, G., Oficina de Publicaciones del Consejo de Estado y Ed. Pueblo y Educación, La Habana, 2005.

7. CAStro Ruz, F.: «Reflexión «Regalo de reyes»», La Habana, 14 de enero de 2008, recuperado de www.cubadebate.cu/reflexiones-fidel/2008/01/14/ regalo-reyes, el 23 octubre de 2010.

8. Cantón Blanco, L.E.: Conferencias de Propiedad y Derechos Reales, Ed. ENSPES, La Habana, 1982.

9. Casullera Arrate, R.: «La Propiedad Personal en nuestra Constitución», en Revista Jurídica No. 10, La Habana, enero-marzo de 1986, pp. 50-67.

10. CRACognA, D.: «La legislación cooperativa en el mundo de hoy», documento presentado en el Seminario de Legislación Cooperativa en Uruguay el 22 de noviembre de 2001, recuperado de http://www.neticoop.org.uy/ article118.html, el 12 de febrero de 2013. 
11. Fernández Peiso, A.: «Estudios jurídicos del cooperativismo», en Anuario del Centro de Investigaciones Jurídicas, 2003, La Habana, pp. 187-210.

12. Fernández Peiso, A.: Lecturas en pro del cooperativismo, ante las imprescindibles transformaciones económicas del socialismo cubano, Universo Sur, Cienfuegos, 2006.

13. Fernández Peiso, A.: «Notas características del marco legal del ambiente cooperativo cubano», en PIÑEIRO HARNECKER, C. (compiladora): Cooperativas y Socialismo. Una mirada desde Cuba, Ed. Caminos, La Habana, 2011.

14. Fernández Peiso, A.: La cooperativa. Bases para su regulación en Cuba, Ed. Ciencias Sociales, La Habana, 2012.

15. GAdeA SOler, E.: Evolución de la legislación cooperativa en España, Consejo Superior de Cooperativas de Euskadi, 1999.

16. Guevara de la Serna, E. (Ché): Apuntes críticos a la economía política, II Edición, Centro de Estudios Ché Guevara, Ocean Press y Ed. Ciencias Sociales, La Habana, 2012.

17. Jiménez Soler, G.: Las empresas de Cuba 1958, Ed. Ciencias Sociales, La Habana, 2004.

18. LezCANo y MAZÓn, A.M.: Constitución de Cuba (con los debates sobre su articulado y transitorias, en la Convención Constituyente), t. II, Ed. Cultural, La Habana, 1941.

19. LÓPEZ CASTILLO, R.: La Ley de Reforma Agraria, con explicaciones prácticas y formulaciones de escritos y acta notarial, Ed. Lex, La Habana, 1959.

20. López labrada, A. y Rodríguez Membrado, E.: "La UBPC: forma de rediseñar la propiedad estatal con gestión cooperativa», en PIÑEIRO HARNECKER, C. (compiladora): Cooperativismo y Socialismo. Una mirada desde Cuba, Ed. Caminos, La Habana, 2012, pp. 337-365.

21. Martínez Hernández, L.: «Raúl: «Lo que hacemos debe ser sometido constantemente a la crítica constructiva por parte de todos»», Periódico Granma, 1 de junio de 2015, recuperado de http://www. granma.cu/cuba/2015-05-31/raul-lo-que-hacemos-debe-ser-sometidoconstantemente-a-la-critica-constructiva-por-parte-de-todos, el 1 de junio de 2015.

22. MiR Pérez, J.: Derecho de Propiedad, t. I, Facultad de Derecho, Universidad de Oriente, ENSPES, 1984.

23. Pichardo, H.: Documentos para la Historia de Cuba, t. IV (Primera Parte), Ed. Ciencias Sociales, La Habana, 1980.

24. PUIG MenESES, Y.: «Autoridades explican nuevas medidas respecto a cooperativas no agropecuarias», La Habana, 9 de agosto de 2017, recuperado en http://www.cubadebate.cu/noticias/2017/08/09/autoridades-explicannuevas-medidas-respecto-a-cooperativas-no-agropecuarias/, el 12 de septiembre de 2017.

25. Rodríguez GARCíA, J.L.: «La perestroika en la economía soviética 19851991», en Revista Internacional Marx Ahora, No. 22, La Habana, 2006, pp. 57-67.

26. Rodríguez Musa, O.: La cooperativa como figura jurídica. Perspectivas constitucionales en Cuba para su aprovechamiento en otros sectores de la 
economía nacional además del agropecuario, Ed. Dykinson S.L., Madrid, 2012.

27. Rodríguez Musa, O.: «La autonomía cooperativa y su expresión jurídica. Una aproximación crítica a su actual implementación legal en Cuba», en Boletín de la Asociación Internacional de Derecho Cooperativo, No. 47, Universidad de Deusto, Bilbao, 2013, pp. 129-155.

28. Rodríguez Musa, O. y Torga Hernández, N.: «La cooperativa de segundo grado: perspectivas para su desarrollo en Cuba», en Boletín de la Asociación Internacional de Derecho Cooperativo, No. 48, Universidad de Deusto, Bilbao, 2014, pp. 81-102.

29. Rodríguez Musa, O.: La constitucionalización de la cooperativa. Una propuesta para su redimensionamiento en Cuba. No. 1 Coletânea IBECOOP, Ed. Vincere Asociados, Brasilia-DF, 2017.

30. VALDÉS LoBÁN, E.: La propiedad socialista y cooperativa en Cuba. Su importancia en el sistema económico, artículo presentado al concurso de la Sociedad Científica de Derecho Económico y Financiero Dr. Ernesto Guerrero Setién, en Biblioteca de la Casa del Jurista en la Provincia de Pinar del Río, 2006.

31. Vega VegA, J.: Derecho Constitucional revolucionario en Cuba, Ed. Ciencias Sociales, La Habana, 1988.

\section{Otros documentos}

1. Autorizadas 16 CoOperativas No AgropeCuARIAS en PRIMER trimestre de 2016, recuperado de http://www.cubadebate.cu/noticias/2016/06/29/autorizadas-16-cooperativas-no-agropecuarias-en-primer-trimestre-de-2016/, el 4 de octubre de 2016.

2. Acta contentiva de la discusión del Proyecto de Ley de Reforma ConstituCIONAL, Décimo Período Ordinario de Sesiones, Tercera Legislatura de la Asamblea Nacional del Poder Popular; días 10, 11 y 12 de julio de 1992.

3. Oficina Nacional de Estadísticas e Información (ONEI): «Listado de cooperativas no agropecuarias con su código, marzo de 2016, recuperado de www.one.cu/ryc/cambian/CNoA.rar, el 4 de octubre de 2016.

4. VII Congreso del Partido Comunista de Cuba: Actualización de los Lineamientos de la Política Económica y Social del Partido y la Revolución para el período 2016-2021, abril de 2016, aprobados además por la Asamblea Nacional del Poder Popular en julio del propio año, recuperado de http:// www.cubadebate.cu/especiales/2016/09/13/vea-el-texto-integro-de-laactualizacion-de-los-lineamientos-para-el-periodo-2016-2021-pdf/, el 2 de octubre de 2016, puntos 15 y 16 principalmente.

5. Castro Ruz, R.: «Discurso pronunciado por el General de Ejército Raúl Castro Ruz, Primer Secretario del Comité Central del Partido Comunista de Cuba y Presidente de los Consejos de Estado y de Ministros, en la clausura del IX Período Ordinario de Sesiones de la VIII Legislatura de la Asamblea Nacional del Poder Popular», La Habana, 14 de julio de 2017, Versiones 
Taquigráficas del Consejo de Estado en Periódico Granma, 15 de julio de 2017.

6. CUBA DeBATE: «Cierran temporalmente el mercado mayorista El Trigal», La Habana, 12 mayo 2016, recuperado de http://www.cubadebate.cu/ noticias/2016/05/12/cierran-el-mercado-mayorista-el-trigal/, el 12 de septiembre de 2017.

7. Ministerio de Finanzas y Precios: «Aprobada extinción de Cooperativa de servicios contables SCENIUS», La Habana, 7 agosto 2017, recuperado en http://www.cubadebate.cu/noticias/2017/08/07/aprobada-extincion-decooperativa-de-servicios-contables-scenius/\#.Wb54j3uR7mM, el 12 de septiembre de 2017.

\section{Legislación}

1. Constitución de la República de Cuba. Gaceta Oficial No. 5 Extraordinaria de 10 de abril de 2019.

2. Constitución de la República de Cuba. Gaceta Oficial de la República de Cuba Edición Especial de 24 de febrero de 1976.

3. Constitución de la República de Cuba. Gaceta Oficial de la República de Cuba No. 464, de 8 de julio de 1940.

4. CÓdIGO DE COMERCIO ESPAÑOL, contenido en el Real Decreto de 22 de agosto de 1885, recuperado de https://www.boe.es/buscar/act.php?id=BOE-A1885-6627, el 18 de junio de 2015.

5. LeY No. 95 DE 2002, "Ley de Cooperativas de Producción Agropecuaria y de Créditos y Servicios». Gaceta Oficial No. 72 Ordinaria de 29 de noviembre de 2002.

6. LEY DE Asociaciones esPAÑola, contenida en el Real Decreto de 13 de junio de 1888, recuperada de https://www.boe.es/buscar/, el 18 de junio de 2015.

7. LEY No. 59 DE 1987, «Código Civil de la República de Cuba», Ministerio de Justicia, La Habana, 1998.

8. LEY No. 599, de 16 de octubre de 1959, sobre «Supresión del Ministerio de Defensa y adscripción de determinados Departamentos a los Órganos que se indican», Gaceta Oficial de 17 de octubre de 1959.

9. Ley de Reforma Agraria, de 17 de mayo de 1959, en Gaceta Oficial de 3 de junio, reproducida en NAVARRETE ACEVEDO, C.R.: Legislación y documentos sobre Derecho Agrario cubano, Facultad de Derecho, Universidad de la Habana, La Habana, 1984.

10. Real DeCreto de 24 de Julio de 1889, por el que se publica el Código Civil español, Agencia Estatal Boletín Oficial del Estado, recuperado de https:// www.boe.es/buscar/pdf/1889/BOE-A-1889-4763-consolidado.pdf, el 16 de junio de 2016.

11. LeY No. 100 DE 23 DE FEBRERO DE 1959, sobre «Creación de Departamentos de Asistencia Educacional y Técnica a las Fuerzas del Ejército Rebelde», Gaceta Oficial No. 33 de 26 de febrero de 1959. 
12. Segunda ley de Reforma Agraria, Gaceta Oficial Extraordinaria No. 1, de 3 de octubre de 1963.

13. Decreto-Ley No. 366/2019. "De las Cooperativas no Agropecuarias», Gaceta Oficial No. 63 Ordinaria de 30 de agosto de 2019.

14. DeCReto-Ley No. 365/2018. "De las Cooperativas Agropecuarias», Gaceta Oficial No. 37 Ordinaria de 24 de mayo de 2019.

15. DeCreto-Ley No. 305/2012. "De las cooperativas no agropecuarias», Gaceta Oficial No. 053 Extraordinaria de 11 de diciembre de 2012 (DEROGADO).

16. Decreto-Ley 142/1993, "Sobre las Unidades Básicas de Producción Cooperativa» (DEROGADO).

17. DeCReto No. 356/2019. "Reglamento de las Cooperativas no Agropecuarias», Gaceta Oficial No. 63 Ordinaria de 30 de agosto de 2019.

18. DeCReto No. 354/2018. "Reglamento del Decreto-Ley de las Cooperativas Agropecuarias», Gaceta Oficial No. 37 Ordinaria de 24 de mayo de 2019.

19. DeCRETO No. 309/2012. «Reglamento de las cooperativas no agropecuarias de primer grado», Gaceta Oficial No. 053 Extraordinaria de 11 de diciembre de 2012 (DEROGADO).

20. Acuerdo del 17 de mayo de 2005, del Comité Ejecutivo del Consejo de Ministros, «Reglamentos Generales de las CPA y las CCS» (DEROGADO).

21. Resolución No. 71 de 1959, en Gaceta Oficial de 30 de octubre de 1959.

22. Resolución No. 462, de 1964 del INRA, Gaceta Oficial No. 8, de 14 de enero de 1964.

23. Resolución No. 5, del Ministerio de Trabajo y Seguridad Social, Gaceta Oficial No. 12 Extraordinaria de 13 de abril de 2016. 


\section{Derechos de autor}

http://dec.revistas.deusto.es/

La revista Deusto Estudios Cooperativos es una revista de acceso abierto lo que significa que es de libre acceso en su integridad inmediatamente después de la publicación de cada número. Se permite su lectura, la búsqueda, descarga, distribución y reutilización legal en cualquier tipo de soporte sólo para fines no comerciales y según lo previsto por la ley; sin la previa autorización de la Editorial (Universidad de Deusto) o el autor, siempre que la obra original sea debidamente citada (número, año, páginas y DOI si procede) y cualquier cambio en el original esté claramente indicado.

\section{Copyright}

The Deusto Journal of Cooperative Studies is an Open Access journal which means that it is free for full and immediate access, reading, search, download, distribution, and lawful reuse in any medium only for non-commercial purposes, without prior permission from the Publisher or the author; provided the original work is properly cited and any changes to the original are clearly indicated. 